Original Research Paper

\title{
Management of Effective Factors in Components' Supply Chain on Losses of Electric Distribution System
}

\author{
${ }^{1}$ M. Esmaeil Honarmand, ${ }^{1}$ M. Sadegh Ghazizadeh and ${ }^{2}$ Ali Kermanshah \\ ${ }^{I}$ Faculty of Electrical Engineering, Shahid Abbaspour Pardis, Shahid Beheshti University, Tehran, Iran \\ ${ }^{2}$ Faculty of Management and Economics, Sharif University, Tehran, Iran
}

\author{
Article history \\ Received: 14-01-2018 \\ Revised: 26-03-2018 \\ Accepted: 20-04-2018 \\ Corresponding Author: \\ M. Esmaeil Honarmand \\ Faculty of Electrical \\ Engineering, Shahid \\ Abbaspour Pardis, Shahid \\ Beheshti University, Tehran, \\ Iran \\ Email: m_e_honar@yahoo.com
}

\begin{abstract}
Restructuring in the electrical distribution utilities, the loss management is one of the critical activities to enhance network efficiency. Accordingly, management of technical loss would be the main target of the asset managers. For loss management, the usual methods of loss calculation and estimation need numerous data which is not used to real networks. In this research, a practical method for loss management is introduced from the viewpoint of components' supply chain to the network. For this purpose, sub-indictors are extracted using focus group for each process of this chain and their probability distribution functions are calculated. Then, permissible values of subindicators are determined by the Delphi method and to calculate new probability functions, the deviation of each process is calculated. Finally, a real network with historical data is used here to study the proposed method. The results illustrate that the most deviation is in the sub-indicator design process about $91 \%$ in the viewpoint losses.
\end{abstract}

Keywords: Energy Loss, Asset Management, Delphi Method, Focus Group, Probability Function

\section{Introduction}

The network loss index is one of the important efficiency indicators in the electric distribution system. This index shows that the overall of electrical distribution utility has an economical viewpoint in the proper utilization of components to supply the customer services (Dashti et al., 2013). Therefore, distribution network operators need to calculate network losses for various purposes, such as assessing the network efficiency; reducing the network losses; calculating the nontechnical losses and decision making about the operation with the optimal configuration (Ababei and Kavasseri, 2011; Yongping, 2011; Monedero et al., 2012). Furthermore, the most of traditional distribution networks are operated to minimum monitoring systems and systems operators are the lack any computational support for network conditions. However, in developing countries, due to energy losses of around $20 \%$, utilities in the electric sector, are currently interested in reducing and managing it in order to be more competitive, since the electricity prices in deregulated markets are related to network losses. Having efficient energy networks is one of the targets to manage network losses. It means that the network losses should be at the decided level of economic efficiency regarding the eliminated amount of energy loss along with reduced air pollution. Accordingly, the elements and measures of loss should be extracted in different parts of the network to manage data and network until the asset manager can choose the proper investment options. Besides, the overall level of managed network losses should be measurable so that it assures for the increase risk due to raise consumer demand is avoided without imposing additional fees on consumers in future. In order to manage the loss reduction plan in the distribution system, it is essential to use the efficient and effective computational tools because, to quantify the network losses in parts of different network, there will be a possibility to manage the network losses.

Generally, the usual ways of loss calculation are categorized into three methods such as to calculate inputs-outputs energy, the estimations of engineering and the sample results. To determine the difference of outputs and inputs of parts the system by the meters, the lack of simultaneous reading is one of the major problems in electric distribution utility. Therefore, due to reasons such as inadequate load data in the 
network, the absence of a reliable database and high cost of data collection, distribution network losses are mostly estimated rather than calculated which these methods are mentioned in various articles.

Wang et al. (2017) presented a novel estimation method of statistical line loss of distribution feeders using the feeder cluster technique and modified eXtreme Gradient Boosting (XGBoost) algorithm. The method is based on the characteristic data of feeders that are collected in the smart power distribution and utilization system. Li et al. (2016) introduced an improved hybrid load flow calculation algorithm and it's simplified for the distribution systems with PV nodes. The calculation accuracy of this method equals to that of NewtonRaphson method and because of the real-number matrix operations, the calculation will be relatively faster. Fei and Lopardo (2012) presented a simple heuristic method for smart distribution system reconfiguration based on the branch-exchange method and single-loop optimization. An integrated analytical approach has been proposed to estimate technical losses of medium voltage distribution network based on the concept of energy flow by Ibrahim et al. (2017). In this reference, a radial MV distribution network is modeled using Representative Feeders (RF) characterized by feeder peak power demand, feeder length, load distribution and load factor to develop the generic analytical technical losses equations. A model based on the stochastic factor of the power covariance matrix which is formed from power load curves has been used to estimate network losses by Shulgin et al. (2012). In this reference, electric power load variation has been presented in the form of a linear combination of some independent random factors and to determine energy losses for a certain time-period. Two formulas of the loss factor for calculating energy losses were improved, based on the Minimum Load Factor (MLF) and the load factor by Fu et al. (2016). Due to the difficulties of estimating losses using maximum demands and the loss factor, Queiroz et al. (2012) have proposed an alternative loss estimation approach that relies on the loss coefficient as the fundamental parameter for describing load variations in loss estimation. All of the mentioned methods presented the estimated loss of current network and then, the management of loss should be investigated by other methods such as network reconfiguration, capacitor placement or installation of distributed generation.

Another method is to use sample results that these have been investigated in some papers. Khoshkolgh Dashtaki and Haghifam (2013) presented a new heuristic method for loss estimation of the lowvoltage sector of a large scale distribution network faced with a scarcity of network data. The mentioned method has used a small part of network data to estimate the total network losses in the LV sector. Madrigal et al. (2015) presented a new methodology for the loss estimation in distribution systems using statistic techniques in order to reduce the amount of information and computing time required for an exact energy loss study. Grigoras and Scarlatache (2013) proposed the use of a new method based on decision trees to estimate the losses in the electric distribution systems. Based on a set of input data processed through clustering techniques a decision tree was built, through which can be estimated with good accuracy losses for one or more distribution feeders An improved stochastic simulation method has been introduced for calculating current dependent energy losses in distribution networks by Mahmoodzadeh et al. (2015). The method is based on power load curves and integrates the stochastic nature of the load curves with power and voltage covariance matrices.

In spite of the fact that the results of these methods have been expressed with high precision, but it is complicated to utilize them on often real networks. Also, there is no link between the distribution utility viewpoint in the issue of network losses and the methods to manage it.

In recent years, the focus on the subject of losses in Iranian distribution companies has been very important so that enhancement of component efficiency and management of network losses has been one of crucial problem of each company. On this base, one of the problems is to extract the effective factors on the losses and to obtain the proper solution by the asset manager for management of losses. The selection and operation method of components in the electric current route is one of the effective elements of the network losses. The inserting of new components such as transformer, cable, wire and so on can affect the losses. Therefore, in this study, the issue of losses is investigated from the viewpoint the new components' supply chain in the distribution system. For this purpose, to extract and to identify the sub-indicators for each process in this supply chain, the performance of processes is monitored and then, the performance deviation is determined by the probability functions and the permissible value of each sub-indicator to select the losses management methods.

\section{Materials and Methods}

\section{Losses and Components' Supply Chain}

The load growth and rising consumer will lead to the development of the network and the inserting of new component in the distribution system. The new components are inserted through various processes so that each process can affect the network losses. The proposed model and required data are shown in Fig. 1 to analyze each process from the viewpoint losses. 


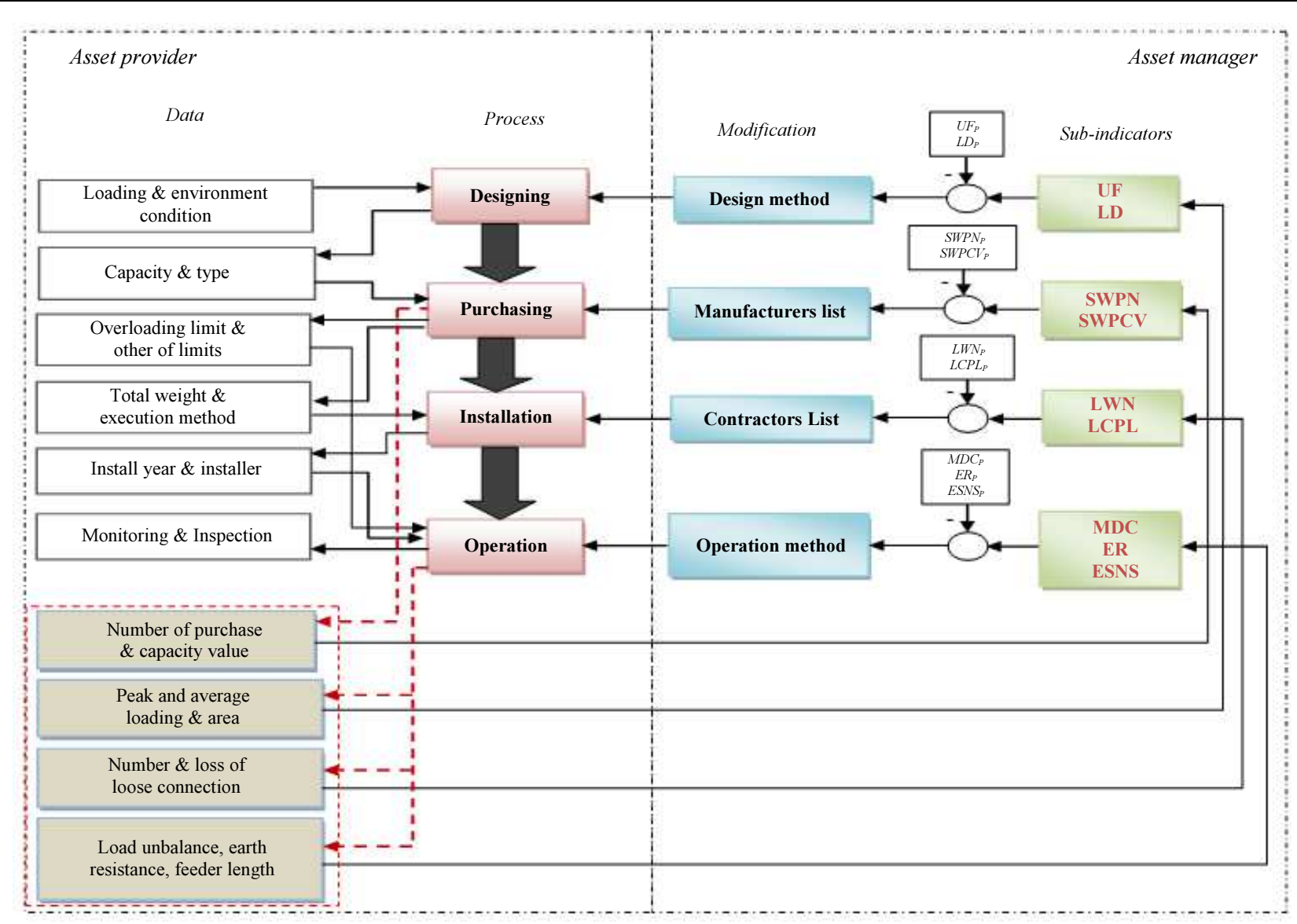

Fig. 1: The proposed model of asset management

From technical losses viewpoint, the flow of electric current from the distribution component will cause the loss in the system. Therefore, if the load prediction is done correctly, the selection and utilization of components will also be appropriate. This action is done by the design process (DE) using input data, such as the loading and environmental conditions. The outputs of this process are the capacity and type of component. On this base, the selection of components is run in three levels, i.e., (1) distribution substation including installation location and transformer capacity, (2) how to supply distribution substation including configuration of medium voltage feeder and (3) the covered area of each substation including configuration of low voltage feeder for customer demand. The Component Purchase (CP) process is initiated after the DE process. In this process according to obtained data, the certifiable component is selected from among given components by manufacturers. The output of this process is divided two parts. One part includes of weight, install year, the method of installation and tests of commissioning that is sent to the Installation and Execution (IE) process. The second part is related to electrical and physical data that is sent to the Operation
(OP) process. After the component installation location was determined, the IE process is initiated. The outputs of this process are the year of installation and the installer that would be transferred to the OP process. To start this process, practically component would be operated in the distribution system and the performance of component should be investigated. Regarding Fig. 1, the model is divided into two parts: The asset provider takes the responsibility of inserting, operating and data gathering of components and the asset manager is responsible for data processing and to provide the reform methods for the improvement of process performance. To survey the process performance from viewpoint loss, an asset manager requires various data from conditions of the network. These data are collected for the candidate sub-indicators of each process. Therefore, data from the $\mathrm{CP}$ and OP processes should be gathered based on the selected sub-indicators as shown in Fig. 1. Each of sub-indicator is compared to a permissible value which is confirmed by the experts, (in Fig 1 is shown as caption "P") and regarding the obtained performance deviation, the revision and improvement in the processes will be decided by the asset manager. 


\section{Methodology of the Proposed Method}

Several data can be measured in a network being operated such as average load, unbalance load, grounding resistance and so on. These data are variable due to some reason, such as variation of load, type of consumer, temperature, location, etc. and can have a random nature. The estimation of network behaviors is one of the missions of network planning and designing in distribution utility so that the proper design can be done regarding technical considerations including network losses. Therefore, the planner thinking can be identified in the network design by tracking these data.

On this base, one of the ways that can track the performance of various parts of the distribution utility in terms of losses is to extract the sub-indicators for the performance of each process and to compare with the permissible values. Therefore, this article tries that the probability distribution function of network behavior obtained using data measured from the distribution network. According to this idea, the effective factors of each process in components' supply chain on network losses are extracted and the probability trend of these is identified. Then, to specify the permissible value of each sub-indicator, the probability distance value is calculated as the performance index of each process. The flowchart of this method is shown in Fig. 2 and the steps are described as follows.

\section{Step 1: The Effective Factors in Losses and the Sub-Indicators}

The proposed method is a statistical approach that the performance of components' supply chain will monitor from the inserting of the component into the network with the detection of the effective factors on network losses. So here, the statistical population is the obtained data based on the effective factors on the losses of each process.

The extraction and selection of the effective factors are done using focus group. Interactive groups are a research technique used to collect data through the interaction of experts on a given topic as defined by the researcher. Indeed, the interaction among the group members is considered as the critical element of data collection in interactive groups (Caridi et al., 2014). This method is particularly useful when access to data is limited and when the researcher is addressing unexplored and emerging phenomena (Liamputtong, 2011). Focus group methodology employs an interviewing technique. The participants are typically a homogeneous group of people who are asked to reflect on a series of questions posed by the interviewer.

To manage the loss in each process of this chain, all relevant activities should be evaluated. Therefore, to form a group of experts in the company, to constitute meetings, to use documentation and to argue in the focus group, the extracted effective factors in the network losses are investigated and the sub-indicator values of each process are introduced.

Then, the required data to measure the process performance are identified using the obtained factors of each process. In order to verify the trend of the process performance, the sub-indicators values are defined and introduced as such to be able to the process modifications can be provided. Therefore, using focus group and to do reviews, the sub-indicators of each process were defined regarding Fig. 1 that the results of discussions in the focus group will be presented as described below.

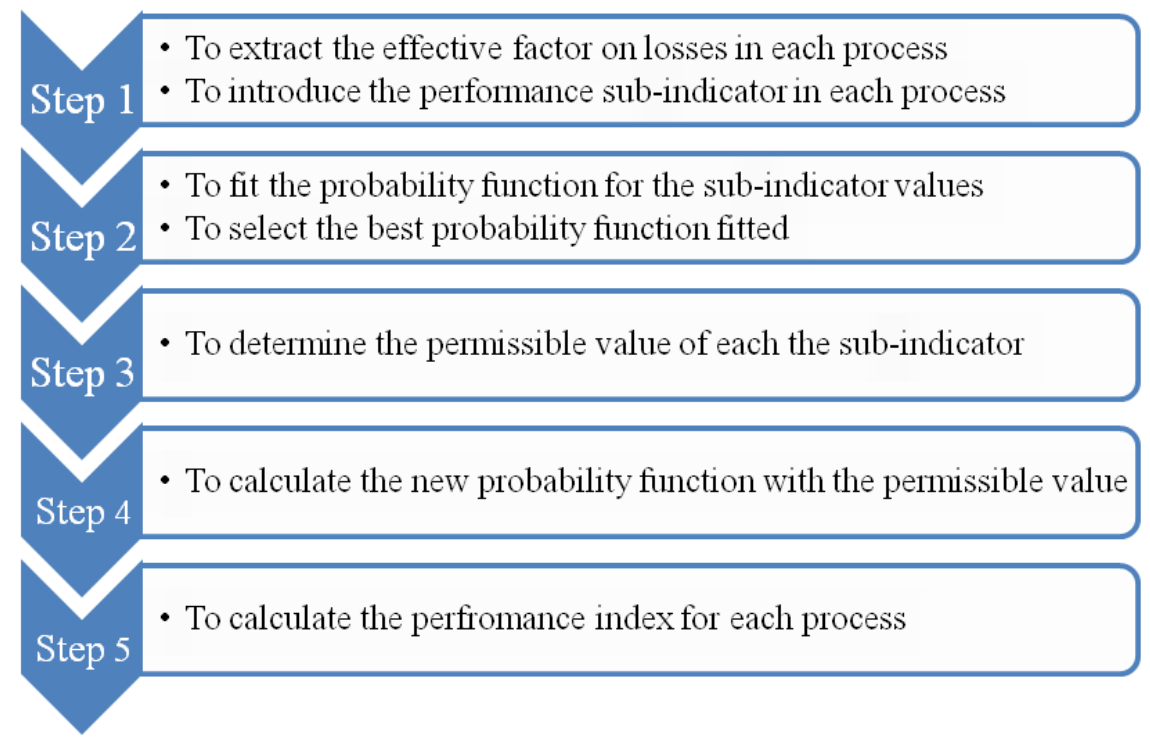

Fig. 2: The proposed method algorithm 
In general, the goal of a network planner is to select the proper component number and capacity and to supply the power to customers. The data such as the load growth, maximum demand and, geographical area must be available for the network design. The installation location and capacity of the transformers and the length, the number and the load value of feeders are determined by these data. Furthermore, the nature of the design is as probable and expectation, so after the charged network, the ampacity of components and the feeder configurations would be determined the precision in design. Therefore, to monitor the DE process, the subindicators such as Utilization Factor (UF) and average Load Density (LD) can be used, typically:

$$
\begin{aligned}
& U F=I_{a v} / I_{M a x} \\
& L D=I_{a v} / A
\end{aligned}
$$

where, $I_{a v}$ and $I_{M a x}$ are averages of current and maximum of current per ampere, respectively and $A$ is the area of the geographic range of the electric load supply per square meters. The sub-indicator of UF can be calculated for the various transformers and feeders and the trend of the design process is investigated using it to select the capacity of transformers and feeders. Furthermore, because there is a relation between the feeder length and the load density, the value of LD is used to investigate the selection of the feeder length and the area of transformers supply.

Also, the components of each design should be purchased based on technical specifications and the designed parameters. If it needs to change the component purchase, this change should be confirmed in the DE process. Sometimes due to reasons such as management decisions, component price and duration of component construction, the changes in the number of buy or the proposed design specifications are made. If these changes in the purchase of a component such as the transformers are considered, it will have a high impact on network losses. So that to purchase a transformer with the higher capacity of the designed parameters, the fixed losses imposed on the system will increase. Therefore, the weighted changes in the purchase are defined as the performance sub-indicator of the $\mathrm{CP}$ process. On this base, two sub-indicators, i.e., the Substituent Purchase Weighted Numbers (SWPN) and the Substituent Purchase Weighted Capacity Value (SWPCV) are considered as the sub-indicators of the $\mathrm{CP}$ process monitoring:

$$
S W P N_{i}=\sum_{i=1}^{k}\left|n_{i} \Delta \Psi_{i}\right| / \sum_{i=1}^{k} \Psi_{i}
$$

$$
S W P C V_{i}=\sum_{i=1}^{k}\left|n_{i} \Delta \Psi_{i}\right| / \sum_{i=1}^{k} n_{i}
$$

where, $n_{i}$ is the number of $i$ th components that differ in design specifications, $\Delta \Psi_{i}$ is the increase or decrease in capacity and $\Psi_{i}$ is the component capacity of purchased. The absolute value of these values can be calculated in a given interval and the trend of the $\mathrm{CP}$ process will be shown according to the proposed options. The weighted sub-indicators would be illustrated the value of change in the effective components of the network losses. If theses the sub-indicators are not zero, they can affect the subindicators of the DE process.

Usually, the impact of the IE process from the losses viewpoint is in the installation method of connections in the electrical current path. Due to the low resistance of connections, this is especially more prominent for the loose connections with high current. If the connections are made as the weak and loose, the high resistance of connections location will cause the losses and increase of the temperature. Commonly to identify the loose connection, a thermo-graphic camera can show the locations of high abnormal temperatures. However, it is difficult to calculate the losses by thermal imaging because it requires to the cross-section and the temperature of connection. Nevertheless, to know the hot spot of the connection location, the connection losses can be estimated. This calculation can be done by a written program by Massey (2009) with a proper approximation. Accordingly, two sub-indicators, i.e., the Loose connection Weighted Numbers (LWN) and the Loose Connection Power Loss (LCPL) are introduced to monitor the performance of the IE process and we have:

$L W N=\sum I_{j} n_{j} / 3 \sum I_{j}$

where $n_{j}$ is the number of identified loose connections and $I_{j}$ is average of single phase current due to the loose connection. These weighted values can be calculated for each the feeder or the transformer.

Network design is often based on balanced load. But in practice, the unbalanced load is inevitable regarding the distribution network structure and the existence of single- or two-phase loads. Whatever the unbalanced load is worse, the network loss is also higher. Therefore, to balance the network load, one of the activities of operator is to avoid increasing the network losses. Also, the proper earth system resistance can obtain a route of the low resistance for the neural current. The low value of earth resistance, not only, can help to better the performance of the protective devices, but also, reduces the network losses that are being operated by reducing displacement of the neutral point. The control and 
inspection of this problem is the task of the OP process. Therefore, to monitor this process, the sub-indicator of the maximum deviation from the mean of current (MDC) and the sub-indicator of earth system resistance value (ER) are used. The different standards introduce different conventional definitions on the meaning of the term current unbalance. According to NEMA-1993, the value of current unbalance can obtain as follows (Yaw-Juen and Ming-Jer, 2000):

$M D C=\frac{\text { Maximum deviation from mean of }\left\{I_{a}, I_{b}, I_{c}\right\}}{\text { mean of }\left\{I_{a}, I_{b}, I_{c}\right\}} \times 100$

where $\left\{I_{a}, I_{b}, I_{c}\right\}$ are currents of each phase. The subindicator of ER alone cannot indicate the overall performance of the OP process. Because it is possible in old distribution networks, the low number of grounding system installed. Therefore, to manage the network losses, the proportion of the grounding system with the network size is one of the network operator tasks.

On this base, the other sub-indicator is defined as the average Earth System Number per Substation (ESNS) that indicates the performance of the OP process and it expressed based on the feeder length of each distribution substation as:

$$
E S N S=E S N / S F L
$$

where, ESN is the installed earth system number and $S F L$ is the sum of the feeder length of each distribution substation. The performance of the OP process will be monitored by these three the sub-indicators.

\section{Step 2: The Probability Distribution of the Sub- Indicators Values}

As mentioned before, to extract and to select the sub-indictors for the monitoring of the performance of processes in the components' supply chain is done in this study. To survey the trend of the obtained subindicators, from the statistical distribution is used regarding the nature of statistical and nonnegative of these values. The various probability distributions are to model the probability behavior of a system that in this study, the probability functions of Weilbull and log-normal have been used as the candidate functions.

The advantage of Weibull function is the ability to provide reasonably accurate analysis and to forecast with extremely small samples. Furthermore, the log-normal distribution that is very similar to the normal distribution, is one of the most common distributions which its parameters can be easily obtained from statistical data. If the random variable logarithm is normally distributed, the random variable is a normal logarithm.
In order to select the most appropriate statistical distribution, the goodness of fit tests is used for a set of the obtained data. The statistical distributions are tools that deal with uncertainty. So, establishing the underlying distribution of a data set (or random variable) is crucial for the correct implementation of some statistical procedures. For this purpose, there are statistical procedures for assessing the underlying distribution of a data set, as the goodness of fit tests. Here among tests of theoretical goodness of fit specialized for small samples, Anderson-Darling (AD) method is interested.

When both statistical distributions are confirmed, the selection of statistical distribution is done by calculation of $p$-value. This value determines the appropriateness of rejecting the null hypothesis in a hypothesis test. The $p$ values range from 0 to 1 and this is the probability of obtaining a test statistic that is at least as extreme as the calculated value if the null hypothesis is true. Thus, the statistical distribution with the greatest $p$-value is selected (Lee and Wenyuwang, 2003).

\section{Step 3: The Assignment of the Permissible Value the Sub-Indicators}

In this step, a permissible value should be selected for each the obtained sub-indicator. The assignment of the permissible value for the sub-indicators can indicate the deviation value from the current case. Generally, the sub-indicators are categorized into two parts. To survey documentations and standards, for a group of them such as UF can assign a permissible value. But another group, the permissible value should be selected regarding network condition, load distribution and geographical location. For these reasons, firstly for each the sub-indicator was suggested a given value. Then, the proposed values are reviewed by the Delphi method and survey of experts in the distribution company. As a data collection method, the Delphi method can fall into the category of both a quantitative and qualitative study. It is useful when the phenomenon under study is complex or when the topic is somehow delicate or the number of members in the focus group is relatively small (Lilija et al., 2011; Galo et al., 2014).

Therefore, the initial table is given to each of the experts individually and, their aspects are collected and analyzed about the assumed values. Then, in a few steps, the summary of comments is sent again to each expert to correct the aspects and ultimately, the final table which contains the proposed permissible value of experts for each the sub-indicators, is provided.

\section{Step 4: The New Probability Function with the Permissible Value}

Each of the probability functions produced for the sub-indicator values in Step 2 has a mean value and a 
standard deviation. The standard deviate shows the distance of the sub-indicator value from the mean value. If the mean value of the probability function is equal to the permissible value obtained in the previous step, this indicates that the value of the sub-indicator is close to the permissible value. On this base, a new probability distribution function is obtained based on the mean value equal to the permissible value. Also, the convergence of company expert's views can be demonstrated by reducing the standard deviation in a specific subindicator. This new probability function will illustrate the data around the permissible value. In this way, a new probability function for each the sub-indicator will calculate by the obtained permissible values.

\section{Step 5: Index of the Performance of Each Process}

The statistical distance in probability is used to quantify the similarity between two statistical distributions. In this step, the concept of statistical distance which actually represents the distance between two the probability distributions can be used as the Index of the performance of each process. To calculate this distance which can be measured by the different methods, the percentage of performance deviation in each process can be expressed from the network losses viewpoint. As defined, assuming that the two statistical distributions obtained are for each the sub-indicator $x$ as $f\left(\mu_{1}, \sigma_{1}, x\right)$ and $g\left(\mu_{2}, \sigma_{2}, x\right)$, where $\mu_{1}$ and $\mu_{2}$ are mean values, $\sigma_{1}$ and $\sigma_{2}$ are standard deviations and $\mu_{2}$ is equal to the permissible value of the sub-indicator, therefore, the defined index for each the sub-indicator is obtained as follows:

$$
\Delta(P, Q)=P I_{k}=\max \left|f\left(\mu_{1}, \sigma_{1}, x\right)-g\left(\mu_{2}, \sigma_{2}, x\right)\right|
$$

where, $P I_{k}$ is defined as the index of $k$ th process. The trend of the network losses into each process is traced and the deviation value is identified.

In general, to determine the probability distance, there are two methods called entropy method and total variation distance that in this study is used to the Hellinger coefficient method. This method is a criterion that quantifies the deviation between the two probability values. Hellinger method presents an intrinsic way to estimate the distances between probability measures independent of the parameters. It is closely related to the total variation distance, but with several advantages. To explain this, let $\mathbf{P}$ and $\mathbf{Q}$ be two probability distributions on a finite sample space $\Omega$, where $\mathbf{P}$ and $\mathbf{Q}$ on $\Omega$ are Ntuples $\left(p_{1}, \ldots p_{N}\right)$ and $\left(q_{1}, \ldots q_{N}\right)$, respectively, satisfying (in)equalities $p_{\alpha} \geq 0, q_{\alpha} \geq 0, \sum_{\alpha} p_{\alpha}=1$ and $\sum_{\alpha} q_{\alpha}=1$. Then, the Hellinger distance (HD) between $\mathrm{P}$ and $\mathrm{Q}$ is defined as (Sengar et al., 2008):

$$
d_{H}^{2}(P, Q)=\frac{1}{2} \sum_{\alpha=1}^{N}\left(\sqrt{p_{\alpha}}-\sqrt{q_{\alpha}}\right)^{2}
$$

The HD satisfied the inequality $0 \leq d_{H}^{2} \leq 1$ and $d_{H}^{2}=0$ when $P=Q$.

The overall algorithm of the proposed method is shown in Fig. 2 and accordingly, the deviation of each process from the permissible value is determined. The benefits of this method can be categorized as follows:

- To analyze the sub-indicators of each process, the management of losses in various parts of the network is identified and planned

- To evaluate each sub-indicator, impact degree of each process can be characterized to manage the network losses

- To review employee mistakes, the asset manager can plan the learning programs

- To assess methods of loss management, various methods such as installation of dispersed generation, reconfiguration, capacitor placement and etc. can be selected and introduced by the asset manager

\section{Results and Discussion}

To conduct numerical studies, the portion of data in Gilan Distribution Company with 35 distribution substations has been chosen that this Company located in the south of Caspian Sea. Based on Fig. 1, the required data such as average load, feeder length, earth system resistance, load unbalance, values related to the loose connections in these substations are gathered and all of the sub-indicators are calculated using these data. In an instance, the calculated sub-indicators of the DE process are shown in Table 1. Due to lack of data in the CP process, the subindicators of this process have not been calculated.

Then, the proper probability function is fitted on these data and according to Table 2, the probability function is selected with the best fit. For example, both probability functions are confirmed for the UF subindicator, but the $p$-value of the Weibull function is bigger, so this function is selected as hatched in the table. In the following, the permissible values of each the sub-indicator were determined using the Delphi method. Accordingly, with the initial assumption for the permissible values, the survey was conducted by a number of experts in the company. In this survey, seven experts were selected from different departments and after collecting forms and analyzing the responses, the survey procedures were repeated three rounds. Eventually, the final results were prepared as Table 3. Afterwards, using the obtained permissible values, new probability functions of each sub-indicator were provided based on the mean value equal to the permissible value and less standard deviation. 
M. Esmaeil Honarmand et al. / American Journal of Applied Sciences 2018, 15 (4): 198.207 DOI: 10.3844/ajassp.2018.198.207

Table 1: The sub-indicators related to the DE process

\begin{tabular}{|c|c|c|c|c|c|}
\hline \multirow[b]{2}{*}{ No. Transformer } & \multicolumn{2}{|c|}{ Sub-indicator } & \multirow{2}{*}{$\begin{array}{l}\text { No. } \\
\text { Transformer }\end{array}$} & \multicolumn{2}{|c|}{ Sub-indicator } \\
\hline & UF & $\mathrm{LD}\left(\mathrm{A} / \mathrm{km}^{2}\right)$ & & UF & $\mathrm{LD}\left(\mathrm{A} / \mathrm{km}^{2}\right)$ \\
\hline 1 & 0.35 & 0.1276 & 19 & 0.36 & 0.0860 \\
\hline 2 & 0.39 & 0.0760 & 20 & 0.44 & 0.1270 \\
\hline 3 & 0.37 & 0.0528 & 21 & 0.56 & 0.0340 \\
\hline 4 & 0.36 & 0.0430 & 22 & 0.46 & 0.0836 \\
\hline 5 & 0.42 & 0.1080 & 23 & 0.41 & 0.1680 \\
\hline 6 & 0.41 & 0.0330 & 24 & 0.44 & 0.1590 \\
\hline 7 & 0.44 & 0.0792 & 25 & 0.44 & 0.0560 \\
\hline 8 & 0.38 & 0.0723 & 26 & 0.43 & 0.0618 \\
\hline 9 & 0.44 & 0.1000 & 27 & 0.55 & 0.0787 \\
\hline 10 & 0.41 & 0.0745 & 28 & 0.40 & 0.0960 \\
\hline 11 & 0.37 & 0.0880 & 29 & 0.32 & 0.1470 \\
\hline 12 & 0.68 & 0.1630 & 30 & 0.45 & 0.1285 \\
\hline 13 & 0.34 & 0.0540 & 31 & 0.42 & 0.1020 \\
\hline 14 & 0.40 & 0.0464 & 32 & 0.47 & 0.0455 \\
\hline 15 & 0.47 & 0.1065 & 33 & 0.37 & 0.0420 \\
\hline 16 & 0.47 & 0.1430 & 34 & 0.25 & 0.0360 \\
\hline 17 & 0.41 & 0.2360 & 35 & 0.48 & 0.0997 \\
\hline 18 & 0.46 & 0.0297 & & & \\
\hline
\end{tabular}

Table 2: The probability function parameters of each the sub-indicator

\begin{tabular}{|c|c|c|c|c|c|c|c|c|c|}
\hline \multirow[b]{3}{*}{ Process } & \multirow[b]{3}{*}{ Sub-indicator } & \multicolumn{4}{|c|}{ Weibull function } & \multicolumn{4}{|c|}{ Log-normal function } \\
\hline & & \multicolumn{2}{|c|}{ Parameter } & \multicolumn{2}{|l|}{ Tests } & \multicolumn{2}{|c|}{ Parameter } & \multicolumn{2}{|c|}{ Tests } \\
\hline & & Shape & Scale & $\mathrm{AD}$ & $p$-value & Location & Scale & $\mathrm{AD}$ & $p$-value \\
\hline \multirow[t]{2}{*}{$\overline{\mathrm{DE}}$} & UF & 22.79 & 0.480 & 3.20 & $<0.01$ & -0.77 & 0.070 & 3.50 & $<0.005$ \\
\hline & LD & 6.35 & 0.088 & 4.90 & $<0.01$ & -2.50 & 0.104 & 2.20 & $<0.005$ \\
\hline \multirow[t]{2}{*}{ IE } & LWN & 3.36 & 0.800 & 3.08 & $<0.01$ & -0.40 & 0.410 & 3.20 & $<0.005$ \\
\hline & LCLP & 1.50 & 0.110 & 1.35 & $<0.01$ & -2.60 & 0.700 & 1.10 & 0.007 \\
\hline \multirow[t]{3}{*}{ OP } & MDC & 2.50 & 4.660 & 1.25 & $<0.01$ & 1.30 & 0.380 & 0.62 & 0.096 \\
\hline & ESNS & 19.50 & 0.018 & 0.61 & 0.108 & -4.06 & 0.070 & 1.50 & $<0.005$ \\
\hline & ER & 1.65 & 3.050 & 4.78 & $<0.01$ & 0.62 & 1.640 & 7.90 & $<0.005$ \\
\hline
\end{tabular}

Table 3: The obtained permissible values of each the sub-indicator

\begin{tabular}{|c|c|c|c|c|c|c|c|}
\hline & \multicolumn{7}{|c|}{ Sub-indicator } \\
\hline & UF & $\mathrm{LD}\left(\mathrm{A} / \mathrm{km}^{2}\right)$ & LWN & $\operatorname{LCLP}(w)$ & MDC (\%) & ESNS (n/Meter) & ER (ohm) \\
\hline Permissible value & 0.7 & 0.15 & 0.2 & 0.06 & 2 & 0.028 & 1 \\
\hline
\end{tabular}

Table 4: The new probability function parameters for the permissible value

\begin{tabular}{llllr}
\hline Process & Sub-indicator & Probability function & Parameters & Values \\
\hline DE & UF & Weibull & Shape & 31.800 \\
& & & Scale & 0.710 \\
& LD & Weibull & Shape & 10.450 \\
& & Scale & 0.156 \\
IE & LWN & Weibull & Shape & 6.340 \\
& & & Scale & 0.210 \\
& LCPL & Weibull & Shape & 2.250 \\
OP & & Scale & 0.070 \\
& MDC & Weibull & Shape & 1.990 \\
& & & Scale & 2.300 \\
& ESNS & Lognormal & Location & -3.570 \\
& & & Scale & 0.067 \\
& ER & Weibull & Shape & 2.210 \\
& & & Scale & 1.130 \\
\hline
\end{tabular}


Table 5: The Hellinger distance for each the sub-indicator

\section{Sub-indicator}

\begin{tabular}{llllllll} 
& UF & LD & LWN & LCLP & MDC & ESNS & ER \\
\hline HD & $35 \%$ & $91 \%$ & $9 \%$ & $18 \%$ & $82 \%$ & $83 \%$ & $9 \%$ \\
\hline
\end{tabular}

The parameters of new probability functions are shown in Table 4. To determine the distance between these two functions, Hellinger distance is calculated which this value represents the deviation of the current value in comparison the permissible value.

The results of these calculations are presented in Table 5. It can be seen from the table that the indicators of the IE process have less diverse than the other two processes. Furthermore, the sub-indicators of the DE process which have a significant impact on the technical losses of the network, have the most deviations so, reviewing the DE process performances should be considered. Among the two sub-indicators of DE process, the LD sub-indicator has the most deviation, therefore, to determine the load density of each transformer, must be reviewed. This issue depends on the location of transformer installation, vicinity of load point, feeder length, etc. In the OP process, the two sub-indicators of MDC and ESNS have the most deviations. The latter subindicator relates to the maintenance of the network and the MDC sub-indicator is dependent on controlling load variations which shows the performance of the OP process requires more control and investigation.

In this study, a process-oriented method is used for extraction of loss factors in electrical distribution network. On this base, the sub-indicators are allocated to each of process from the viewpoint loss so that the performance of processes is shown by these. Unlike other methods, the estimation or evaluation of loss is not performed by the proposed method rather to monitor the performances and to extract the effective factors, the actions or the decisions of the asset manager would be represented in the loss management. Furthermore, because this article is based on new the components' supply chain, so every change in the network structure and component is monitored from the viewpoint of loss and the practical procedures are considered for each process.

While in other methods, the operation-base performance of the network is presented in the loss modeling and is assumed that the performances of other processes in the utility are probably perfect and the operation department should be responsible only for the network loss. This problem is caused which the effective factors of the loss are identified in the network and management of loss is considered only based on the change in view of the network operation.

\section{Conclusion}

This paper has focused on the loss management from the components' supply chain viewpoint into the distribution network. Accordingly, the model of the supply chain has been introduced and the sub-indicators for each process of this chain have been determined from losses viewpoint. Regarding the probable nature of the network behavior, the probability functions have been used for the tracing, investigation and monitoring of the process performance. Then, to define the permissible value of each sub-indicator, new probability functions have been obtained and the probability distance of these two functions was calculated as the performance deviation value of each process. Ultimately, to assess the deviation value via the asset manager, the improvement methods of each process will be proposed for asset management. Indeed, the proposed method can be employed to control and to revise the effective factors on losses through to monitor the different processes in the components' supply chain.

The results of this paper demonstrate that among the various processes of the supply chain, the DE process has the most deviation from the defined permissible values. This matter will need to revise the method of the network designer and planner from viewpoint losses. Also, regarding the performance deviation on the OP process, the activities of operators should be reformed from this perspective.

Obviously, there is a range of requisite relevant studies which are proposed here as the subjects of future research. These can deal with the subjects such as detecting the effective factors in other processes of electrical distribution system, the impacts of cost-related and the investigation of process-based non technical loss.

\section{Acknowledgement}

The authors are grateful to Gilan Electric Power Distribution Company (EPDC), Gilan, Iran for their support in gathering of data.

\section{Author's Contributions}

M. Esmaeil Honarmand: Participated in all experiments, organized the study and contributed to the writing of the manuscript.

M. Sadegh Ghazizadeh: Designed the research plan and coordinated the data-analysis and contributed to the writing of the manuscript.

Ali Kermanshah: Participated in all experiments and assisted data analysis.

\section{Ethics}

This article has not been published before and the data listed are in accordance with the results of our team's research. We ensure there is no ethical involvement. 


\section{References}

Ababei, C. and R. Kavasseri, 2011. Efficient network reconfiguration using minimum cost maximum flow-based branch exchanges and random walksbased loss estimations. IEEE Trans. Power Syst., 26: 30-37. DOI: 10.1109/TPWRS.2010.2052076

Caridi, M., A. Moretton, A. Perego and A. Tumino, 2014. The benefits of supply chain visibility: A value assessment model. Int. J. Product. Econom., 151: 1-19. DOI: 10.1016/j.ijpe.2013.12.025

Dashti, R., S. Yousefi and M. Parsa Modhaddam, 2013. Comprehensive efficiency evaluation model for electrical distribution system considering social and urban factors. Energy, 60: 53-61. DOI: $10.1016 /$ j.energy.2013.07.042

Fei, D. and K.A. Lopardo, 2012. A simple heuristic method for smart distribution system reconfiguration. Proceedings of the IEEE Energytech, May 29-31, IEEE Xplore Press, Cleveland, OH, USA, pp: 1-6. DOI: 10.1109/EnergyTech.2012.6304698

$\mathrm{Fu}, \mathrm{X}$., H. Chen, R. Cai and P. Xuan, 2016. Improved LSF method for loss estimation and its application in DG allocation. IET Gener. Transm. Distrib, 10: 2512-2519. DOI: 10.1049/iet-gtd.2015.1471

Galo, J.J.M., M.N.Q. Macedo, L.A.L. Almeida and A.C.C. Lima, 2014. Criteria for smart grid deployment in Brazil by applying the Delphi method. Energy, 70: 605-611. DOI: 10.1016/j.energy.2014.04.033

Grigoras, G. and F. Scarlatache, 2013. Energy losses estimation in electrical distribution networks with a decision trees-based algorithm. Proceedings of the 8th International Symposium on Advanced Topics in Electrical Engineering, May 23-25, IEEE Xplore Press, Bucharest, Romania, pp: 1-4. DOI: 10.1109/ATEE.2013.6563522

Ibrahim, K.A., M.T. Au, C.K. Gan and J.H. Tang, 2017. System wide MV distribution network technical losses estimation based on reference feeder and energy flow model. Int. J. Electr. Power Energy Syst., 93: 440-450.

DOI: $10.1016 /$ j.ijepes.2017.06.011

Khoshkolgh Dashtaki, A. and M.R. Haghifam, 2013. A new loss estimation method in limited data electric distribution networks. IEEE Trans. Power Delivery, 28: 2194-2200.

DOI: 10.1109/TPWRD.2013.2273103

Lee, E.T. and J. Wenyuwang, 2003. Statistical Methods for Survival Data Analysis. 3rd Edn., John Wiley and Sons, ISBN-10: 0471369977, pp: 534.

Li, H., Y. Jin, A. Zhang, X. Shen and C. Li et al., 2016. An improved hybrid load flow calculation algorithm for weakly-meshed power distribution system. Int. J. Electrical Power Energy Syst., 74: 437-445.

DOI: 10.1016/j.ijepes.2015.08.004
Liamputtong, P., 2011. Focus Group Methodology: Principle and Practice. 1st Edn., SAGE, Los Angeles, ISBN-10: 1446242803, pp: 224.

Lilija, K.K., K. Laakso and J. Palomaki, 2011. Using the Delphi method. Proceedings of the Energy Smart World, (ESW' 11), pp: 1-10.

Madrigal, M., J. Jesus Rico and L. Uzcategui, 2015. Estimation of technical energy losses in electrical distribution systems. IEEE Latin Am. Trans., 13: 3310-3316. DOI: 10.1109/TLA.2015.7387236

Mahmoodzadeh, Z., N. Ghanbari, A. Mehrizi-Sani and M. Ehsan, 2015. Energy loss estimation in distribution networks using stochastic simulation. Proceedings of the IEEE Power and Energy Society General Meeting, Jul. 26-30, IEEE Xplore Press, Denver, CO, USA, pp: 1-5. DOI: 10.1109/PESGM.2015.7286219

Massey, L., 2009. Correcting small loose connections, big power savings. Proceedings InfraMation.

Monedero, I., F. Biscarri, C. Leon, J.I. Guerrero and J. Biscarri et al., 2012. Detection of frauds and other non-technical losses in a power utility using Pearson coefficient, Bayesian networks and decision trees. Electrical Power Energy Syst., 32: 90-98. DOI: 10.1016/j.ijepes.2011.09.009

Queiroz, L.M.O., M.A. Roselli, C. Cavellucci and C. Lyra, 2012. Energy losses estimation in power distribution systems. IEEE Trans. Power Syst., 27: 1879-1887. DOI: 10.1109/TPWRS.2012.2188107

Sengar, H., H. Wang, D. Wijesekera and S. Jajodia, 2008. Detecting VoIP floods using the hellinger distance. IEEE Trans. Parallel Distributed Syst., 19: 794-805. DOI: 10.1109/TPDS.2007.70786

Shulgin, I.V., A.A. Gerasimenko and S.Q. Zhoua, 2012. Modified stochastic estimation of load dependent energy losses in electric distribution networks. Int. J. Electrical Power Energy Syst., 43: 325-332.

DOI: $10.1016 /$ j.ijepes.2012.03.034

Wang, S., P. Dong and Y. Tian, 2017. A novel method of statistical line loss estimation for distribution feeders based on feeder cluster and modified XGBoost. Energies, 10: 2-17. DOI: 10.3390/en10122067

Yaw-Juen, W. and Y. Ming-Jer, 2000. Probabilistic modeling of three-phase voltage unbalance caused by load fluctuations. Proceedings of the IEEE Power Engineering Society Winter Meeting, Jan. 23-27, IEEE Xplore Press, Singapore, pp: 2588-2593. DOI: 10.1109/PESW.2000.847290

Yongping, Z., 2011. Line losses management system of distribution networks based on local area network. Proceedings of the Asia-Pacific Power and Energy Engineering Conference, Mar. 25-28, IEEE Xplore Press, Wuhan, China, pp: 1-3.

DOI: 10.1109/APPEEC.2011.5748744 